\title{
A new analytic solution of complex Langevin differential equations
}

\author{
Rabha W. Ibrahim \\ IEEE RAS Malaysia Chapter, Kuala Lumpur, Malaysia
}

\begin{abstract}
Purpose - In this study, the authors introduce a solvability of special type of Langevin differential equations (LDEs) in virtue of geometric function theory. The analytic solutions of the LDEs are considered by utilizing the Caratheodory functions joining the subordination concept. A class of Caratheodory functions involving special functions gives the upper bound solution.

Design/methodology/approach - The methodology is based on the geometric function theory.

Findings - The authors present a new analytic function for a class of complex LDEs.

Originality/value - The authors introduced a new class of complex differential equation, presented a new technique to indicate the analytic solution and used some special functions.
\end{abstract}

Keywords Subordination and superordination, Analytic function, Univalent function, Open unit disk, Majorization

Paper type Research paper

\section{Introduction}

Langevin differential equation (LDE) is one of the most important differential equation in mathematical sciences, including fluid, Brownian motion, thermal and wavelet studies. It investigated wildly in view of various types of geometric, stochastic and analysis studies (see for example references [1-5]). An arbitrary model of LDEs is studied in [6-8] including analytic solutions. The existence and stability of a class of LDEs with two HilferKatugampola fractional derivatives is investigated in [9]. Moreover, the existence of LDE is illustrated suggesting different types of geometry [10,11].

LDEs of a complex variable are applied to simulate special types of polymer and nanomaterials, including the conduct of the polymers [12]. Based on this priority of LDEs of a complex variable, we aim to study this class analytically. The technique of the geometric function theory is used recently by Ibrahim and Baleanu [13] to determine the fractal solution. They utilized different notions such as the subordination and super-ordination, majorization, Caratheodory functions, convex functions and special functions (see [14-16]).

Here, we discuss the upper bound solution of LDEs of a complex variable in feature of geometric function theory. We illustrated a list of conditions that implies a univalent result in $\cup$ (the unit disk). The mechanism of our proof is considered utilizing the Caratheodory functions joining the subordination concept. A class of Caratheodory functions involving special functions gives the upper bound solution.

\section{JEL Classification - 30C55, 30C45.}

(C) Rabha W. Ibrahim. Published in Arab Journal of Mathematical Sciences. Published by Emerald Publishing Limited. This article is published under the Creative Commons Attribution (CC BY 4.0) licence. Anyone may reproduce, distribute, translate and create derivative works of this article (for both commercial and non-commercial purposes), subject to full attribution to the original publication and authors. The full terms of this licence may be seen at http://creativecommons.org/licences/by/4.0/ legalcode

The author wishes to introduce many thanks to the respected reviewers for their kind comments and the editorial board for their advice.
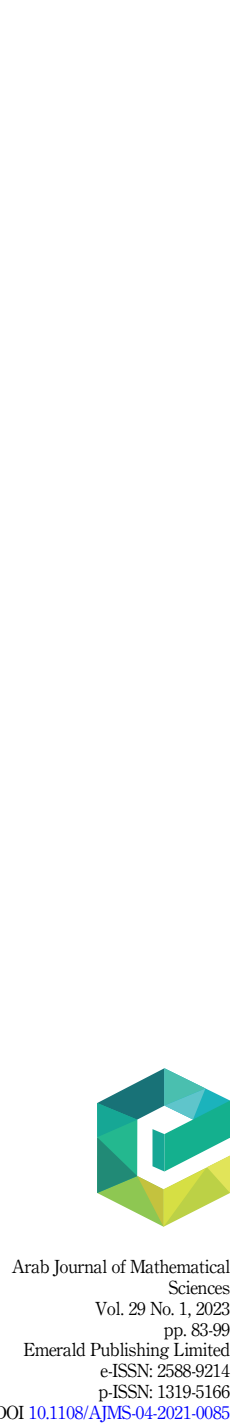
AJMS

29,1

84

\section{Complex Langevin differential equations}

The LDE of a complex variable can be realized by the next formula [17].

$$
\chi^{\prime \prime}(z)+\alpha \chi^{\prime}(z)=F(\chi(z)), \quad z \in \mathbb{C},
$$

where $\alpha>0$ indicates the oscillation coefficient, and $F$ is the noise factor. To study the geometric properties of Eq.(2.1), we consider $z \in U=\{z \in \mathbb{C}:|z|<1\}$, and $\chi(z)$ is a normalized function satisfying the expansion $\chi(z)=z+\sum_{n=2}^{\infty} \chi z^{n}$. Rearrange Eqn (2.1) with complex coefficient, then the homogeneous formula is given by

$$
\Psi(z):=\varsigma(z)\left(\frac{z^{2} \chi^{\prime \prime}(z)}{\chi(z)}\right)+\left(\frac{z \chi^{\prime}(z)}{\chi(z)}\right), \quad z \in \cup,
$$

where $\varsigma(z)$ is analytic function in $\cup$. It is clear that $\Psi(0)=1$, for all $\varsigma(z) \in \cup$ (see the following example).

Example 2.1. Let data given by

(1) $\chi(z)=z /(1-z), \varsigma(z)=z$, then we have $\Psi(z)=1+z+3 z^{2}+5 z^{3}+7 z^{4}+9 z^{5}+O\left(z^{6}\right)$;

(2) $\chi(z)=z /(1-z)^{2}, \varsigma(z)=z$, then we get $\Psi(z)=1+2 z+6 z^{2}+12 z^{3}+18 z^{4}+24 z^{5}+O\left(z^{6}\right)$;

(3) $\varsigma(z)=1-z$ and $\chi(z)=z /(1-z)$, then we obtain $\Psi(z)=1+3 z+3 z^{2}+3 z^{3}+3 z^{4}$ $+3 z^{5}+O\left(z^{6}\right)$

(4) $\varsigma(z)=1$ and $\chi(z)=z /(1-z)$, then we obtain $\Psi(z)=1+3 z+5 z^{2}+7 z^{3}+9 z^{4}+11 z^{5}$ $+O\left(z^{6}\right)$.

We denote by $\mathcal{P}(A, B)$, the class of functions

$$
\rho(z)=\frac{1+A w(z)}{1+B w(z)}<\frac{1+A z}{1+B z},
$$

where $w$ satisfies $w(0)=0$ and $|w(z)|<1$; and $-1 \leq B<A \leq 1$, then $\mathcal{P}(A, B) \subset \mathcal{P}\left(\frac{1-A}{1-B}\right)$ is the Janowski class. Next, we define a class of analytic functions.

Definition 2.2. The function $\chi(z)=z+\sum_{n=2}^{\infty} \chi_{n} z^{n}, z \in \cup$ is in $\mathbf{M}_{\varsigma}(\rho)$ if and only if

$$
\begin{gathered}
\Psi(z)=\varsigma(z)\left(\frac{z^{2} \chi^{\prime \prime}(z)}{\chi(z)}\right)+\left(\frac{z \chi^{\prime}(z)}{\chi(z)}\right)<\rho(z) . \\
\left(z \in \cup, \rho(0)=1, \rho^{\prime}(0)>1, \varsigma \in U, \varsigma(z) \in U\right) .
\end{gathered}
$$

Now consider starlike function as follows:

$$
\rho_{e}(z)=\frac{z}{e^{z}-1}=1-\frac{z}{2}+\frac{z^{2}}{12}-\frac{z^{4}}{720}+\ldots
$$

and a convex function

$$
\varrho_{e}(z):=1 / \rho_{e}(z)=1+\frac{z}{2}+\frac{z^{2}}{6}+\frac{z^{3}}{24}+\frac{z^{4}}{120}+\ldots
$$


(see[18]-P415). We note here that the coefficients are approximating to the Bernoulli numbers such that

$$
\Re\left(\frac{e^{\xi z}-1}{\xi z}\right) \geq \frac{1}{2}, \quad 0<\xi \leq 1.793 \ldots
$$

Hence, $\Re\left(\frac{e^{\xi z}-1}{\xi z}\right) \geq 1 / \rho_{e}(-1)=\frac{1}{2}$.

Our design is generated by the Caratheodory functions, which are operated in [19]. In this situation, we establish the necessary conditions of the joining bounds of $\Psi(z)$ consuming a Caratheodory function. Note that, when $\varsigma(z)$ is a constant, the class $\mathbf{M}_{\varsigma}(\rho)$ reduces to the well-known class in [20].

\subsection{Geometric properties}

Some geometric properties are illustrated as follows:

Proposition 2.3. Consider the functional $\Psi(z)$ such that $p(z)=z \chi(z)^{\prime} / \chi(z)$. Then $\chi(z)$ is starlike in $\cup$, whenever $\varsigma(z)=z, \mathfrak{R}(z)>0$ and

$$
-\sqrt{(3)}(\Re(z))<\Im(z)<\sqrt{(3)}(\Re(z)) .
$$

Proof. Suppose the functional

$$
\Psi(z)=\varsigma(z)\left(\frac{z^{2} \chi^{\prime \prime}(z)}{\chi(z)}\right)+\left(\frac{z \chi^{\prime}(z)}{\chi(z)}\right) .
$$

Let $p(z)=z \chi(z)^{\prime} / \chi(z)$, then

$$
(z)\left(\frac{z \chi(z)^{\prime \prime}}{\chi(z)}\right)=z\left(z p^{\prime}(z)\right)+z p^{2}(z)-z p(z)
$$

yields that

$$
\Psi(z)=z\left(z p^{\prime}(z)\right)+z p^{2}(z)+(1-z) p(z), \quad z \in \cup .
$$

By [18]-Example 2.4m, we have $A(z)=z, B(z)=z, C(z)=1-z$ and $D(z)=0$, where the assumptions imply that $\mathfrak{R}(z)>0$ we get the conclusion

$$
\begin{aligned}
& \mathfrak{R}\left[A(z) z p^{\prime}(z)+B(z) p^{2}(z)+C(z) p(z)+D(z)\right] \\
& =\Re\left[z\left(z p^{\prime}(z)\right)+z p^{2}(z)+(1-z) p(z)\right] \\
& >0 \Rightarrow \mathfrak{R}(\not(z))>0 \text {. }
\end{aligned}
$$

Corresponding to the above conclusion, we indicate that $\chi(z)$ is starlike.

Proposition 2.3 can be generated for $\Re(\varsigma(z))>0$ as follows:

Proposition 2.4. Consider the functional $\Psi(z)$ such that $p(z)=z \chi(z)^{\prime} / \chi(z)$. Then $\chi(z)$ is starlike in $\cup$, whenever $\Re(\varsigma(z))>0$ and

$$
[\mathfrak{s}(\varsigma(z))]^{2}<3(\Re(\varsigma(z)))^{2} .
$$

\section{Proposition 2.5. (Integral existence result)}

Consider the functional $\Psi(z)=p(z) \cdot q(z)$, where $p(z)=z \chi(z)^{\prime} / \chi(z)$ and

$$
q(z)=\varsigma(z)\left(1+\frac{z \chi^{\prime \prime}(z)}{\chi^{\prime}(z)}\right) .
$$


AJMS

29,1

If $\varsigma(0)=1$ and the subordination

$$
\left(\alpha_{1}+\alpha_{2}\right)+\frac{z q^{\prime}(z)}{q(z)}<\left(\alpha_{1}+\alpha_{2}\right)\left(\frac{1+z}{1-z}\right)+\frac{2 z}{1-z^{2}}
$$

holds such that $\alpha_{1}+\alpha_{2}=\beta_{1}+\beta_{2}>0$, then the integral

$$
V(z):=\left(\frac{\beta_{1}+\beta_{2}}{z^{\left(\beta_{2}\right)} p(z)} \int_{0}^{z} \zeta^{\alpha_{1}+\alpha_{2}-1} q(\zeta) d \zeta\right)^{1 /\left(\beta_{1}\right)}
$$

satisfies the following conclusion

$$
\left(V(z) \in \wedge, V(z) / z \neq 0, \Re\left(\beta_{1} \frac{z V^{\prime}(z)}{V(z)}+\frac{z q^{\prime}(z)}{q(z)}+\beta_{2}\right)>0\right) .
$$

Proof. Consider $\Psi(z)$ with $\varsigma(z)=1$, then a computation implies that

$$
\Psi(z)=\left(\frac{z \chi^{\prime}(z)}{\chi(z)}\right)\left(1+\frac{z \chi^{\prime \prime}(z)}{\chi^{\prime}(z)}\right)
$$

Since, $p(0)=1$ and $q(0)=1$ with $\Psi(z)=p(z) q(z) \neq 0$ for some $z_{0} \in U$. Then in view of [18]-Theorem 2.5c, we have the desired conclusion.

Proposition 2.6. Consider the functional $\Psi(z)=p(z) \cdot q(z)$, where $p(z)=z \chi(z)^{\prime} / \chi(z)$ and

$$
q(z)=\left(1+\frac{z \chi^{\prime \prime}(z)}{\chi^{\prime}(z)}\right), \varsigma(0)=1
$$

If one of the following facts is indicated

(1) $\mathfrak{R}\left(\frac{z q^{\prime}(z)}{q(z)}\right)+\delta>0$;

(2) $\left|\mathfrak{s}\left(\frac{z q^{\prime}(z)}{q(z)}+\delta\right)\right|<\sqrt{1+2 \delta}, \delta>0$;

(3) $\left|\frac{z q^{\prime}(z)}{q(z)}\right|<\delta+1, \delta>0$,

then the integral

$$
W(z):=\left(\frac{\delta}{z^{\delta-1} p(z)} \int_{0}^{z} \zeta^{\delta-1} q(\zeta) d \zeta\right)
$$

satisfies the conclusion

$$
\left(W(z) \in \wedge, W(z) / z \neq 0, \mathfrak{R}\left(\frac{z W^{\prime}(z)}{W(z)}+\frac{z q^{\prime}(z)}{q(z)}+1\right)>0\right) .
$$

Proof. A computation implies that

$$
\Psi(z)=\varsigma(z)\left(1+\frac{z \chi^{\prime \prime}(z)}{\chi^{\prime}(z)}\right)\left(\frac{z \chi^{\prime}(z)}{\chi(z)}\right), \varsigma(z) \in U .
$$


Since $p(0)=1$ and $q(0)=1$ with $\Psi(z)=p(z) q(z) \neq 0$ for some $z_{0} \in U$. Then in view of [18]-Corollary 2.5c.1, we get the desired conclusion.

Next example shows the integral existence result of the convex Koebe function $z /(1-z)$. We confirm that the integral formula is also convex because it is majorized by $z /(1-z)$.

Example 2.7. Let $\chi(z)=z /(1-z)$ and $\varsigma(z)=1$, then we have $p(z)=1 /(1-z)$ and

$$
\begin{aligned}
q(z) & =\left(1+\frac{z \chi^{\prime \prime}(z)}{\chi^{\prime}(z)}\right) \\
& =\frac{1+z}{1-z} \\
& =1+2 z^{2}+2 z^{3}+2 z^{4}+2 z^{5}+O\left(z^{6}\right) .
\end{aligned}
$$

Thus, we obtain

$$
\left|\frac{z q^{\prime}(z)}{q(z)}\right|<\delta+1 \Leftrightarrow \delta>0, \frac{\delta}{\delta+2}<\Re(z)<\delta .
$$

Then by letting $\delta=1$, we conclude that the integral existence result satisfies

$$
\begin{aligned}
W(z) & =\frac{\delta}{z^{\delta-1} p(z)} \int_{0}^{z} \zeta^{\delta-1} q(\zeta) d \zeta \\
& =(1-z) \int_{0}^{z}\left(\frac{1+\zeta}{1-\zeta}\right) d \zeta \\
& =(z-1)(z+2 \log (1-z)) \\
& =z-z^{3} / 3-z^{4} / 6-z^{5} / 10-z^{6} / 15+O\left(z^{7}\right) \in \wedge,
\end{aligned}
$$

which achieves all indicated facts in Proposition 2.5. Since the coefficient bounds of $W(z)$ are motorized by the coefficient bounds of $\chi(z)$, then we conclude that $W(z)$ is convex. Moreover, the iteration of the integral existence theorem of a convex function $(\chi(z))$ remains convex in the open unit disk $(W(W \ldots(W(z))))$. As a conclusion, this example provides a chain of analytic convex solutions of LDEs in $U$. Next remark shows the important relation of $W(z)$ with the function of nephroid plane curve. This leads to use $W(z)$ as an official formula in a nephroid plane curve instead of using parametric functions. Moreover, Proposition 2.5 implies a positive real solution of LDEs; for example, by assuming $\beta_{1}=1, \beta_{2}=0$, we get $\Re\left(\frac{z W^{\prime}(z)}{W(z)}+\frac{z q^{\prime}(z)}{q(z)}\right)>0$ because $W(z)$ and $q(z)$ are starlike in $\cup$ satisfying $\Re\left(\frac{z W^{\prime}(z)}{W(z)}\right)>0$ and $\mathfrak{R}\left(\frac{z q^{\prime}(z)}{q(z)}\right)>0$. As a comparison with recent methods, our method provides in spite of an analytic solution, the strategy of the existing integral formula involving the analytic solution is still analytic in $\cup$. Note that this solution is univalent in $\cup$. All recent techniques provided an analytic solution without geometric presentations. Our method describes the analytic solution and its integration geometrically.

Remark 2.8. It is well known that the function $\omega(z)=1+z-z^{3} / 3$ (see Figure 1) translates the unit circle onto a 2-cusped curve tilled nephroid satisfying $\left((w-1)^{2}+v^{2}-4 / 9\right)^{3}-$ $4 / 3 v^{2}=0$. The functional $\varpi(z):=1+W(z)$ can be expanded by (see Figure 2 ) 

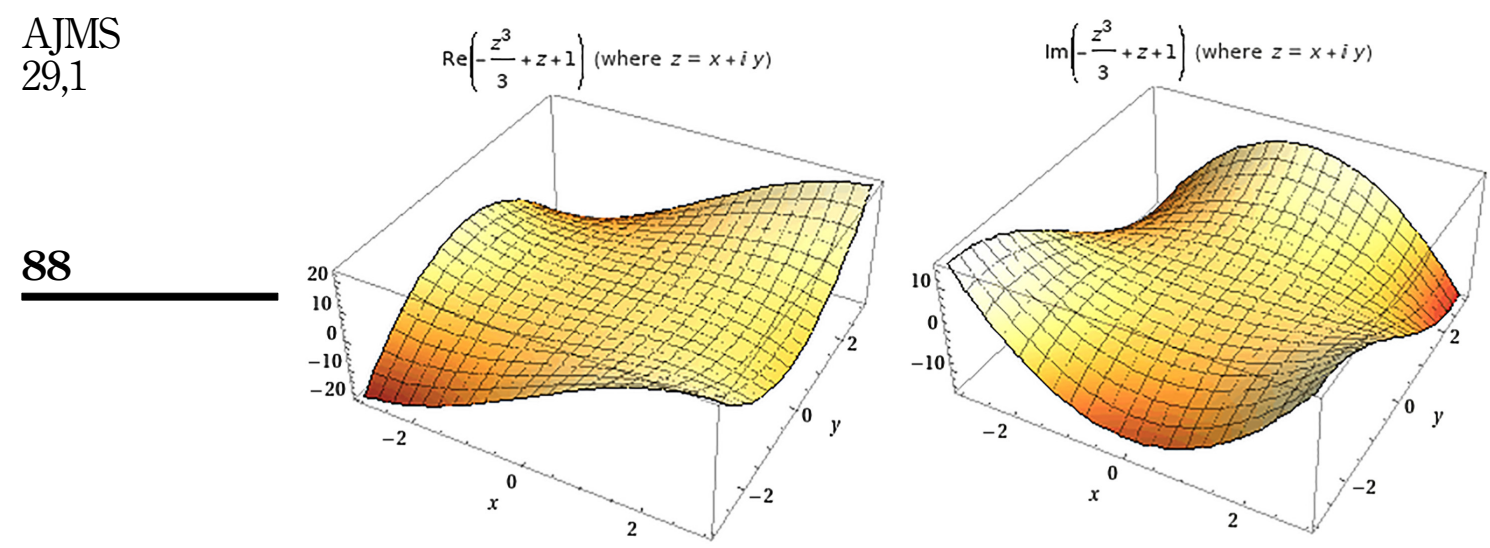

Figure 1.

The plot of $\omega(z)$
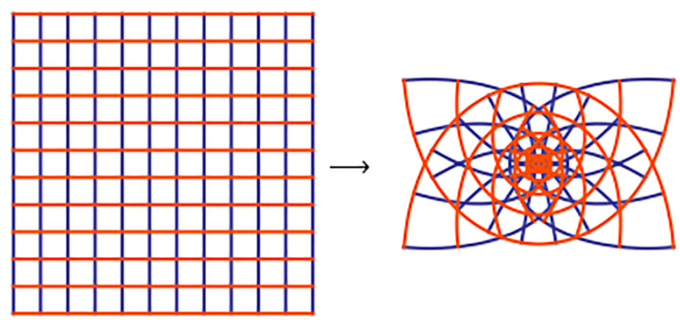

$\left.\operatorname{Re}\left(\frac{1}{30}\left(-2 z^{6}-3 z^{5}-5 z^{4}-10 z^{3}+30 z+30\right)\right)\right]\left(\right.$ where $z=x+i . \quad \operatorname{Im}\left(\frac{1}{30}\left(-2 z^{6}-3 z^{5}-5 z^{4}-10 z^{3}+30 z+30\right)\right)($ where $z=x+i)$

Figure 2.

The plot of $\varpi(z)$
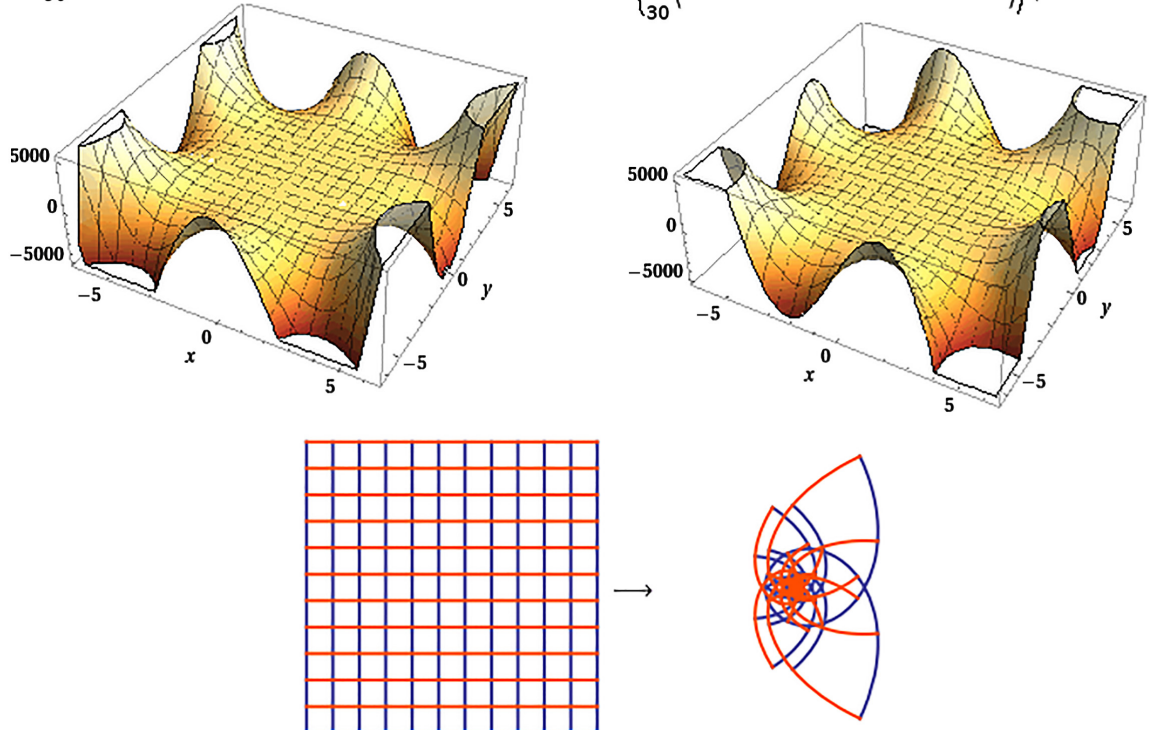


$$
\varpi(z)=\omega(z)-z^{4} / 6-z^{5} / 10-z^{6} / 15+O\left(z^{7}\right) .
$$

We shall use $\varpi(z)$ to define some interesting classes of analytic functions.

\section{Computations}

This section deals with some computational outcomes utilizing a sigmoid function. Note that a sigmoid function is bounded analytic in convex complex domain (see Figure 3 ).

Theorem 3.1. Suppose that $\chi \in \wedge$ achieves the inequality

$$
1+\mu\left(\frac{z \Psi^{\prime}(z)}{[\Psi(z)]^{k}}\right)<\frac{2}{1+e^{-z}}, \quad k=0,1,2,
$$

where $\Psi(z)=\varsigma(z)\left(\frac{z^{2} \chi^{\prime \prime}(z)}{\chi(z)}\right)+\left(\frac{z \chi^{\prime}(z)}{\chi(z)}\right), \quad z \in \cup$. Then

$$
\Psi(z)<\rho_{e}(z)=\frac{z}{e^{z}-1}, z \in U
$$

when $\mu \geq \max \mu_{k}$.
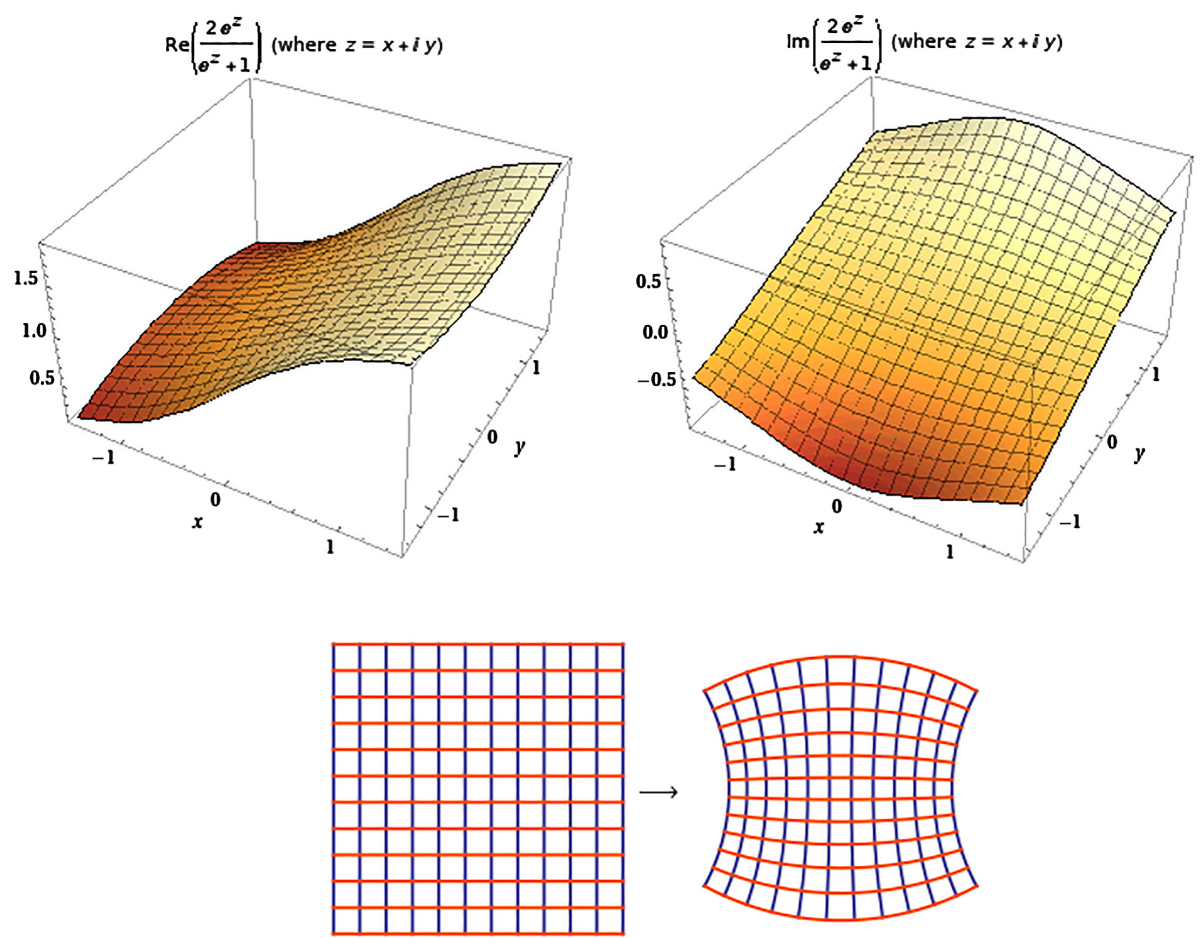

Figure 3. The plot of sigmoid function $s(z)=\frac{2}{1+e^{-2}}$, where its max is at $\frac{2 e}{1+e^{\prime}}$ and its $\min$ is at $\frac{2}{1+e}$ 
AJMS
29,1

90
(1) $\quad \max \mu_{0}=\max \left\{\frac{35}{72}(e-1), \frac{(35(e-1))}{(72(e-2))}\right\} \approx 1.1628$.

(2) $\quad \max \mu_{1}$

$=\max \left\{\frac{-35}{(72(\log (e-1)-1))}, \frac{35}{(72 \log (e-1))}\right\}$

$\approx 1.1$.

(3) $\quad \max \mu_{2}$

$=\max \left\{\frac{35 e}{72}, \frac{35}{72(e-2)}\right\}$

$\approx 1.321$.

Proof. Case [A]: assume that $k=0 \Rightarrow 1+\mu\left(z \Psi^{\prime}(z)\right)<\frac{2}{1+e^{-2}}$.

Formulate a function $X_{\mu}: \cup \rightarrow \mathbb{C}$ by the structure

$$
X_{\mu}(z)=1+\frac{1}{\mu}\left(\frac{z}{2}-\frac{z^{3}}{72}+\frac{z^{5}}{1200}+\ldots\right),
$$

where

$$
\begin{gathered}
\int_{0}^{z} \frac{e^{\zeta}-1}{\zeta\left(1+e^{\zeta}\right)}= \\
\frac{z}{2}-\frac{z^{3}}{72}+\frac{z^{5}}{1200}-\frac{\left(17 z^{7}\right)}{282240}+\frac{\left(31 z^{9}\right)}{6531840}-\frac{\left(691 z^{11}\right)}{1756339200}+O\left(z^{13}\right)+\text { constant } .
\end{gathered}
$$

It is clear that $X_{\mu}(z)$ is an analytic solution of

$$
1+\mu\left(z X_{\mu}^{\prime}(z)\right)=\frac{2}{1+e^{-z}}, \quad z \in \mathrm{U} .
$$

Consider the functional $\mathfrak{l}(z):=\mu\left(z X_{\mu}^{\prime}(z)\right)=\frac{2}{1+e^{-2}}-1=\frac{e^{-z}-1}{e^{-2}+1}$, which is starlike in $\cup[19]$. This implies that for $\mathfrak{S}(z):=\mathfrak{H}(z)+1$, we have

$$
\Re\left(\frac{z \mathfrak{H}^{\prime}(z)}{\mathfrak{H}(z)}\right)=\mathfrak{R}\left(\frac{z \mathfrak{W}^{\prime}(z)}{\mathfrak{H}(z)}\right)>0 .
$$

Consequently, Miller-Mocanu Lemma [18] indicates that

$$
1+\mu\left(z \Psi^{\prime}(z)\right)<1+\mu\left(z X_{\mu}^{\prime}(z)\right) \Rightarrow \Psi(z)<X_{\mu}(z) .
$$

To end this organization, we aim to show that $X_{\mu}(z)<\rho_{e}(z)$. Obviously, $X_{\mu}(z)$ increases in $(-1,1)$ for some $\mu$ that is fulfilling 


$$
\begin{aligned}
& 1+\frac{\left(-1+\frac{2 e}{e-1}+\log (2)+\log (e-1)-\log (3 e-1)\right)}{\mu} \\
& \approx 1+\frac{-2.8}{\mu} \\
& \leq X_{\mu}(-1) \leq X_{\mu}(1) \\
& \approx 1+\frac{(1+e)}{(e-1)}+2 \tanh ^{(-1)} \frac{(1+e)}{(3-5 e)} / \mu \\
& \approx 1+\frac{1.4306}{\mu} .
\end{aligned}
$$

Since the function $\rho_{e}(z)$ fulfills the relation

$$
(e-1)^{-1} \leq \mathfrak{R}\left(\rho_{e}(z)\right) \approx 1-\frac{\cos (\vartheta)}{2}+\sum_{n=1}^{\infty} \frac{\beta_{2 n} \cos (2 n \vartheta)}{(2 n) !} \leq e(e-1)^{-1},
$$

then consequently, we arrive at the inequality

$$
(e-1)^{-1} \leq X_{\mu}(-1) \leq X_{\mu}(1) \leq e(e-1)^{-1}
$$

whenever $\mu$ satisfies

$$
\mu \geq \max \mu_{0}=\max \left\{\frac{35}{72}(e-1), \frac{(35(e-1))}{(72(e-2))}\right\} \approx 1.1628 .
$$

Consequently, we obtain

$$
X_{\mu}(z)<\frac{z}{e^{z}-1} \Rightarrow \Psi(z)<\frac{z}{e^{z}-1} .
$$

Case [B]: consume the case $k=1 \Rightarrow 1+\mu\left(\frac{z \Psi^{\prime}(z)}{\Psi(z)}\right)<\frac{2}{1+e^{-2}}$.

Formulate a function $Y_{\mu}: \cup \rightarrow \mathbb{C}$ by the equation

$$
Y_{\mu}(z)=\exp \left(\frac{1}{\mu}\left(\frac{z}{2}-\frac{z^{3}}{72}+\frac{z^{5}}{1200}+\ldots\right)\right) .
$$

Clearly, we have a solution $Y_{\mu}(z)\left(Y_{\mu}(0)=1\right)$ of the differential equation

$$
1+\mu\left(\frac{z Y_{\mu}^{\prime}(z)}{Y_{\mu}(z)}\right)=\frac{2}{1+e^{-z}}, \quad z \in \mathrm{U} .
$$

Consider the starlike function $\mathfrak{S}(z)=2 /\left(1+e^{-z}\right)-1$ then the functional $\mathfrak{S}(z)=\mathbb{G}(z)+1$ implies $\mathfrak{R}\left(\frac{z \mathfrak{S}^{\prime}(z)}{\mathfrak{G}(z)}\right)=\mathfrak{R}\left(\frac{z \mathfrak{S}^{\prime}(z)}{\mathfrak{S}(z)}\right)>0$. Again Miller-Mocanu Lemma gives 
AJMS
29,1

$$
1+\mu\left(\frac{z \Psi^{\prime}(z)}{\Psi(z)}\right)<1+\mu\left(\frac{z Y_{\mu}^{\prime}(z)}{Y_{\mu}(z)}\right) \Rightarrow \Psi(z)<Y_{\mu}(z) .
$$

Proceeding, we have

$$
(e-1)^{-1} \leq Y_{\mu}(-1) \leq Y_{\mu}(1) \leq e(e-1)^{-1}
$$

if $\mu$ when

$$
\begin{aligned}
\mu \geq & \max \mu_{1} \\
& =\max \left\{\frac{-35}{(72(\log (e-1)-1))}, \frac{35}{(72 \log (e-1))}\right\} \\
& \approx 1.1 .
\end{aligned}
$$

This implies

$$
Y_{\mu}(z)<\frac{z}{e^{z}-1} \Rightarrow \Psi(z)<\frac{z}{e^{z}-1}, \quad z \in \cup .
$$

Case [C]: assume that $k=2 \Rightarrow 1+\mu\left(\frac{z \Psi^{\prime}(z)}{\Psi^{2}(z)}\right)<\frac{2}{1+e^{-2}}$.
The function

$$
D_{\mu}(z)=\left(1-\frac{1}{\mu}\left(\frac{z}{2}-\frac{z^{3}}{72}+\frac{z^{5}}{1200}+\ldots\right)\right)^{-1} .
$$

is a solution for the differential equation

$$
1+\mu\left(\frac{z D_{\mu}^{\prime}(z)}{D_{\mu}^{2}(z)}\right)=\frac{2}{1+e^{-z}} .
$$

As a conclusion, Miller-Mocanu Lemma yields

$$
1+\mu\left(\frac{z \Psi^{\prime}(z)}{\Psi^{2}(z)}\right)<1+\mu\left(\frac{z D_{\mu}^{\prime}(z)}{D_{\mu}^{2}(z)}\right) \Rightarrow \Psi(z)<D_{\mu}(z) .
$$

Accordingly, we have

$$
(e-1)^{-1} \leq D_{\mu}(-1) \leq D_{\mu}(1) \leq e(e-1)^{-1}
$$

if $\mu_{2}$ recognizes the upper and lower bounds

$$
\begin{aligned}
\mu & \geq \max \mu_{2} \\
& =\max \left\{\frac{35 e}{72}, \frac{35}{72(e-2)}\right\} \\
& \approx 1.321 .
\end{aligned}
$$

This indicates the relation

$$
D_{\mu}(z)<\frac{z}{e^{z}-1} \Rightarrow \Psi(z)<\frac{z}{e^{z}-1}, \quad z \in \cup .
$$


Theorem 3.1 can be extended to functions in $\mathcal{P}$. We omit the proof.

Theorem 3.2. Let $p \in \mathcal{P}$ achieving the inequality

$$
1+\mu\left(\frac{z p^{\prime}(z)}{[p(z)]^{k}}\right)<\frac{2}{1+e^{-z}}, \quad k=0,1,2, \mu \geq 1.321 .
$$

Then

$$
p(z)<\rho_{e}(z)=\frac{z}{e^{z}-1}, z \in \cup .
$$

We deal with the function $\varrho_{e}(z)=\frac{e^{z}-1}{z}$, which is convex univalent.

Theorem 3.3. Consider the hypotheses of Theorem 3.1. Then

$$
\Psi(z)<\varrho_{e}(z) .
$$

when $v \geq \max v_{k}$.

$$
\max v_{0}=\max \left\{\frac{35}{(72(e-2))}, \frac{(35 e)}{72}\right\} \approx 1.321
$$

$$
\max v_{1}=\max \left\{\frac{35}{(72 \log (e-1))}, \frac{-35}{(72(\log (e-1)-1))}\right\} \approx 1.12
$$

$$
\max v_{2}=\max \left\{\frac{(35(e-1))}{(72(e-2))}, \frac{35}{72(e-1)}\right\} \approx 1.162
$$

Proof. Clearly, we have $(e-1) / e \leq \mathfrak{R}\left(Q_{e}(z)\right) \leq e-1$. Consequently, we obtain $(e-1) /$ $e \leq X_{v}(-1) \leq X_{v}(1) \leq e-1$ whenever $v$ satisfies

$$
\begin{aligned}
v & \geq \max v_{0} \\
& =\max v_{0}=\max \left\{\frac{35}{(72(e-2))}, \frac{(35 e)}{72}\right\} \\
& \approx 1.321 .
\end{aligned}
$$

This implies the relation

$$
X_{v}(z)<\frac{e^{z}-1}{z} \Rightarrow \Psi(z)<\frac{e^{z}-1}{z} .
$$

In the same manner, we get

$$
\begin{aligned}
v & \geq \max v_{1} \\
& =\max \left\{\frac{35}{(72 \log (e-1))}, \frac{-35}{(72(\log (e-1)-1))}\right\} \\
& \approx 1.12
\end{aligned}
$$


AJMS

29,1

94

Consequently, we obtain

$$
Y_{v}(z)<\frac{e^{z}-1}{z} \Rightarrow \Psi(z)<\frac{e^{z}-1}{z} .
$$

Finally, we have

$$
\begin{aligned}
v & \geq \max v_{2} \\
& =\max \left\{\frac{(35(e-1))}{(72(e-2))}, \frac{35}{72(e-1)}\right\} \\
& \approx 1.162 .
\end{aligned}
$$

This implies that the result

$$
D_{v}(z)<\frac{e^{z}-1}{z} \Rightarrow \Psi(z)<\frac{e^{z}-1}{z} .
$$

Theorem 3.3 can be generalized by utilizing $p \in \mathcal{P}$. The proof is similar to the above proof.

Theorem 3.4. Suppose that $p \in \mathcal{P}$ satisfies

$$
1+\mu\left(\frac{z p^{\prime}(z)}{[p(z)]^{k}}\right)<\frac{2}{1+e^{-z}}, \quad k=0,1,2, \mu \geq 1.321 .
$$

Then

$$
p(z)<\varrho_{e}(z)=\frac{e^{z}-1}{z}, z \in \cup .
$$

Next result indicates the upper bound:

$$
J(z)=\frac{1+A z}{1+B z},(-1 \leq B<A \leq 1),
$$

(bi-linear transformation) which is starlike function with positive real part.

Theorem 3.5. Consider one of the following inequalities

(1) $1+\ell\left(z \Psi^{\prime}(z)\right)<\sqrt{z+1}, \ell \geq \max \left\{\ell_{0}, \ell_{1}\right\}$, where

$$
\ell_{0}=\frac{2(0.22599 B+0.22599)}{(A-B)}, \quad B+1 \neq 0, A-B \neq 0 ;
$$

and

$$
\ell_{1}=\frac{2((B-1)(\log (2)-1))}{(A-B)}, B-1 \neq 0, A-B \neq 0 .
$$

(2) $1+\ell\left(z \frac{\Psi^{\prime}(z)}{\Psi(z)}\right)<\sqrt{z+1}, \ell \geq \max \left\{\ell_{2}, \ell_{3}\right\}$, where

$$
\ell_{2}=\frac{2(i(-1+\sqrt{(2)}+\log (2)-\log (1+\sqrt{(2)})))}{2 \pi n-i \log \left(\frac{B+1}{A+1}\right)},
$$




$$
\left(B+1 \neq 0, A+1 \neq 0, \log \left(\frac{B+1}{A+1}\right)+2 \pi n i \neq 0\right)
$$

and

$$
\ell_{3}=\frac{-2(i(\log (2)-1))}{2 \pi n-i \log \left(\frac{A-1}{B-1}\right)} ; \log \left(\frac{A-1}{B-1}\right)+2 i \pi n \neq 0, A \neq 1, B \neq 1
$$

(3) $1+\ell\left(z \frac{\Psi^{\prime}(z)}{\Psi^{2}(z)}\right)<\sqrt{z+1}, \ell \geq \max \left\{\ell_{4}, \ell_{5}\right\}$, where

$$
\begin{gathered}
\ell_{4}=\frac{2(0.225987 A+0.225987)}{(A-B)}, B+1 \neq 0, A \neq B ; \\
\ell_{5}=\frac{2((A-1)(\log (2)-1))}{(A-B)}, \quad B-1 \neq 0 A \neq B .
\end{gathered}
$$

Then $\Psi(z)<\frac{1+A z}{1+B z},(-1 \leq B<A \leq 1)$.

Proof. Case [A]: Let $k=0 \Rightarrow 1+\ell\left(z \Psi^{\prime}(z)\right)<\sqrt{z+1}$.

Define a function $F_{\ell}: \cup \rightarrow \mathbb{C}$ admitting the structure

$$
F_{\ell}(z)=1+\frac{2}{\ell}(\sqrt{z+1}-\log (1+\sqrt{z+1})-1+\log (2)) .
$$

It is clear that $F_{\ell}(z)$ is analytic in $\cup$ satisfying $F_{\ell}(0)=1$, and it is a solution of the differential equation

$$
1+\ell\left(z F_{\ell}^{\prime}(z)\right)=\sqrt{z+1}, \quad z \in \cup .
$$

Therefore, this yields $\mathfrak{U}(z):=\ell\left(z F_{\ell}^{\prime}(z)\right)=\sqrt{z+1}-1$ is starlike in $\cup$. So in view of MillerMocanu Lemma, we get

$$
1+\mu\left(z \Psi^{\prime}(z)\right)<1+\ell z F_{\ell}^{\prime}(z) \Rightarrow \Psi(z)<F_{\ell}(z) .
$$

To end this argument, we must show that $F_{\ell}(z)<J(z)$. Evidently, $F_{\ell}(z)$ increases in $(-1,1)$, such that $F_{\ell}(-1) \leq F_{\ell}(1)$. Since

$$
\frac{1-A}{1-B} \leq F_{\ell}(-1) \leq F_{\ell}(1) \leq \frac{1+A}{1+B}
$$

whenever $\ell \geq \max \left\{\ell_{0}, \ell_{1}\right\}$ where

$$
\ell_{0}=\frac{2(0.22599 B-0.22599)}{(A-B)}, \quad B+1 \neq 0, A-B \neq 0 ;
$$

and

$$
\ell_{1}=\frac{2((B-1)(\log (2)-1))}{(A-B)}, B-1 \neq 0, A-B \neq 0 .
$$


AJMS

29,1

Consequently, we obtain

$$
F_{\ell}(z)<J(z) \Rightarrow \Psi(z)<J(z), \quad z \in U .
$$

Case [B]: assume that $k=1 \Rightarrow 1+\ell\left(\frac{z \Psi^{\prime}(z)}{\Psi(z)}\right)<\sqrt{z+1}$.

The function

$$
S_{\ell}(z)=\exp \left(\frac{2}{\ell}(\sqrt{z+1}-\log (1+\sqrt{z+1})-1+\log (2))\right) .
$$

is a solution of the differential equation

$$
1+\ell\left(\frac{z S_{\ell}^{\prime}(z)}{S_{\ell}(z)}\right)=\sqrt{z+1}, \quad z \in \mathrm{U} .
$$

Then again, in virtue of the Miller-Mocanu Lemma, we arrive at

$$
1+\mu\left(\frac{z \Psi^{\prime}(z)}{\Psi(z)}\right)<1+\ell\left(\frac{z S_{\ell}^{\prime}(z)}{S_{\ell}(z)}\right) \Rightarrow \Psi(z)<S_{\ell}(z) .
$$

Thus, we obtain

$$
\frac{1-A}{1-B} \leq S_{\ell}(-1) \leq S_{\ell}(1) \leq \frac{1+A}{1+B}
$$

whenever $\ell \geq \max \left\{\ell_{2}, \ell_{3}\right\}$ where

$$
\begin{gathered}
\ell_{2}=\frac{(2 i(-1+\sqrt{(2)}+\log (2)-\log (1+\sqrt{(2)})))}{2 \pi n-i \log \left(\frac{B+1}{A+1}\right)}, \\
\left(B+1 \neq 0, A+1 \neq 0, \log \left(\frac{B+1}{A+1}\right)+2 \pi n i \neq 0\right)
\end{gathered}
$$

and

$$
\ell_{3}=\frac{-2(i(\log (2)-1))}{2 \pi n-i \log \left(\frac{A-1}{B-1}\right)} ; \log \left(\frac{A-1}{B-1}\right)+2 i \pi n \neq 0, A \neq 1, B \neq 1 .
$$

This indicates the relations

$$
S_{\ell}(z)<J(z) \Rightarrow \Psi(z)<J(z), \quad z \in U .
$$

Case[C]: suppose that $k=2 \Rightarrow 1+\ell\left(\frac{z \Psi^{\prime}(z)}{\Psi^{2}(z)}\right)<\sqrt{z+1}$.
The function

$$
Q_{\ell}(z)=\left(1-\frac{2}{\ell}(\sqrt{z+1}-\log (1+\sqrt{z+1})-1+\log (2))\right)^{-1}
$$

is a solution of the differential equation 


$$
1+\mu\left(\frac{z Q_{\ell}^{\prime}(z)}{Q_{\ell}(z)}\right)=\sqrt{z+1}, \quad z \in \cup .
$$

Clearly, Miller-Mocanu Lemma implies

$$
1+\ell\left(\frac{z \Psi^{\prime}(z)}{\Psi^{2}(z)}\right)<1+\ell\left(\frac{z Q_{\ell}^{\prime}(z)}{Q_{\ell}^{2}(z)}\right) \Rightarrow \Psi(z)<Q_{\ell}(z) .
$$

Accordingly, we have

$$
\frac{1-A}{1-B} \leq Q_{\ell}(-1) \leq Q_{\ell}(1) \leq \frac{1+A}{1+B}
$$

if $\ell_{2}$ recognizes the upper and lower bounds

$$
\begin{gathered}
\ell_{4}=\frac{2(0.225987 A+0.225987)}{(A-B)}, B+1 \neq 0, A \neq B ; \\
\ell_{5}=\frac{2((A-1)(\log (2)-1))}{(A-B)}, \quad B-1 \neq 0 A \neq B .
\end{gathered}
$$

This brings that

$$
Q_{\ell}(z)<J(z) \Rightarrow \Psi(z)<J(z)
$$

Note that, in Theorem 3.5, we can replace $\Psi(z)$ by the general function $p(z) \in \mathcal{P}$ to get $p(z)<J(z)$. We advance to extant the upper bound result of Eq. (2.3) by the singular function $\lambda(z)=1+\sin (z), z \in \cup$, where it is with positive real part. The proof is quite similar to Theorem 3.5; therefore, we omit it.

Theorem 3.6. Consider one of the following inequalities

(1) $1+\tau\left(z \Psi^{\prime}(z)\right)<\sqrt{z+1}, \tau \geq \max \left\{\tau_{0}, \tau_{1}\right\}$, where

$$
\tau_{0}=2(-1+\sqrt{(2)}+\log (2)-\log (1+\sqrt{(2)})) \csc (1) ;
$$

and

$$
\tau_{1}=2(\log (2)-1)(-\csc (1))
$$

(2) $1+\tau\left(z \frac{\Psi^{\prime}(z)}{\Psi(z)}\right)<\sqrt{z+1}, \tau \geq \max \left\{\tau_{2}, \tau_{3}\right\}$, where

$$
\tau_{2}=\frac{2(-1+\sqrt{(2)}+\log (2)-\log (1+\sqrt{(2)}))}{\log (1+\sin (1))},
$$

and

$$
\tau_{3}=\frac{2(\log (2)-1)}{\log (1-\sin (1))} .
$$


AJMS

29,1
(3) $1+\tau\left(z \frac{\Psi^{\prime}(z)}{\Psi^{2}(z)}\right)<\sqrt{z+1}, \tau \geq \max \left\{\tau_{4}, \tau_{5}\right\}$, where

$$
\begin{gathered}
\tau_{4}=2(-1+\sqrt{(2)}+\log (2)-\log (1+\sqrt{(2)}))(1+\csc (1)) \\
\tau_{5}=2(\log (2)-1)(-(\csc (1)-1)) .
\end{gathered}
$$

Then $\Psi(z)<1+\sin (z), z \in \cup$.

By using the technique of Theorem 3.5, we have the following result using $\varpi(z)$.

Theorem 3.7. Consider one of the following inequalities

Then

(1) $1+\ell\left(z \Psi^{\prime}(z)\right)<\sqrt{z+1}, \ell \geq \max \left\{\ell_{0}, \ell_{1}\right\}=\left\{\frac{-10}{4(\log (2)-1)}, \frac{21(\log (27)-6)}{(\log (8)-4)}\right\} \approx 2.816$;

(2) $1+\ell\left(z \frac{\Psi^{\prime}(z)}{\Psi(z)}\right)<\sqrt{z+1}, \ell \geq \max \left\{\ell_{2}, \ell_{3}\right\}=\left\{\frac{2(1-\log (2))}{\log (5)}, \frac{2(\log (3)-2)}{(-1-\log (2)+\log (3))}\right\} \approx 3$;

(3) $1+\ell\left(z \frac{\Psi^{\prime}(z)}{\Psi^{2}(z)}\right)<\sqrt{z+1}, \ell \geq \max \left\{\ell_{4}, \ell_{5}\right\}=\left\{2 \frac{-(4(\log (3)-2))}{(\log (16)-3)}, \frac{2(2-\log (3))}{(\log (2)-5)}\right\} \approx-0.4$.

$$
\Psi(z)<\varpi(z)=1+z-z^{3} / 3-z^{4} / 6-z^{5} / 10-z^{6} / 15+O\left(z^{7}\right), \quad z \in \cup .
$$

In Theorems 3.6 and 3.7 , one can replace $\Psi(z)$ by $p(z)$ to get more general results $p(z)<1+\sin (z)$ and $p(z)<\varpi(z)$ respectively.

\section{Conclusion}

From above, we conclude that LDEs can be recognized in terms of a complex variable $z \in U$. We illustrated a list of sufficient conditions for the existence of holomorphic univalent solutions. Our next study will be considered for a generalized class of analytic functions in the open unit disk.

\section{References}

1. Nadler B, Schuss Z, Singer A, Robert S, Eisenberg. Ionic diffusion through confined geometries: from Langevin equations to partial differential equations. J Phys Condensed Matter. 2004; 16(22): S2153.

2. Wax N, editor. Selected papers on noise and stochastic processes. New York, NY: Dover; 1954.

3. Mazo R. Brownian motion: fluctuations, dynamics and applications. Oxford: Oxford Univ. Press; 2002.

4. Coffey W.T. Yu P. Kalmykov, Waldron J.T. The Langevin Equation, seconded. Singapore: World Scientific; 2004.

5. Zwanzig R. Non equilibrium statistical mechanics. New York, NY: Oxford University Press; 2001.

6. Jeon JH, Metzler R. Fractional Brownian motion and motion governed by the fractional Langevin equation in confined geometries. Phys Rev E. 2010; 81(2): 021103.

7. Ahmadova A, Mahmudov NI. Langevin differential equations with general fractional orders and their applications to electric circuit theory. J Comput Appl Math; 388(2021): 113299.

8. Mahmudov NI., Huseynov IT., Aliev NA., Aliev FA. Analytical approach to a class of BagleyTorvik equations. TWMS J Pure Applied Math. 2020; 11(2): 238-258.

9. Ibrahim RW., Harikrishnan S, Kanagarajan K. Existence and stability of Langevin equations with two Hilfer-Katugampola fractional derivatives. Stud. Univ. Babes-Bolyai Math. 2018; 63(3): 291-302. 
10. Feng $\mathrm{C}$, Zhao H, Zhong J. Existence of geometric ergodic periodic measures of stochastic differential equations. arXiv preprint arXiv:1904.08091; 2019.

11. Thieu TK., Adrian M. Solvability of a Coupled Nonlinear System of Skorohod-Like Stochastic Differential Equations Modeling Active-Passive Pedestrians Dynamics Through a Heterogeneous Domain and Fire. arXiv preprint arXiv:2006.00232; 2020.

12. Lennon EM, Mohler GO, Ceniceros HD, Garcia-Cervera CJ, Fredrickson GH. Numerical solutions of the complex Langevin equations in polymer field theory. Multiscale Model Simulation. 2008; 6(4): $1347-1370$.

13. Ibrahim RW., Dumitru B. Analytic solution of the Langevin differential equations dominated by a multibrot fractal set. Fractal and Fractional. 2021; 5(2): 50.

14. Ibrahim RW., Elobaid RM., Obaiys SJ. Symmetric conformable fractional derivative of complex variables. Mathematics. 2020; 8(3): 363.

15. Ibrahim RW., Elobaid RM., Obaiys SJ. Generalized Briot-Bouquet differential equation based on new differential operator with complex connections. Axioms. 2020; 9(2): 42.

16. Ibrahim RW., Elobaid RM., Obaiys SJ. On subclasses of analytic functions based on a quantum symmetric conformable differential operator with application. Adv Diff Eq. 2020; 2020(1): 1-14.

17. Vanden-Eijnden E, Ciccotti G. Second-order integrators for Langevin equations with holonomic constraints. Chem Phys Lett. 2006; 429(1-3): 310-316.

18. Miller SS., Mocanu PT. Differential subordinations: theory and applications. New York and Basel CRC Press; 2000.

19. Wani, Ahmad L, Swaminathan A. Differential subordinations for starlike functions associated with A nephroid domain; 2019. arXivarXiv-1912.

20. Lee SK, Ravichandran V., Supramaniam S. Initial coefficients of biunivalent functions. Abstract and applied analysis, 2014. Hindawi; 2014.

\section{Corresponding author}

Rabha W. Ibrahim can be contacted at: rabhaibrahim@yahoo.com
Langevin differential equations 